LAWRENCE LIVERMORE NATIONAL LABORATORY

\title{
Analytic Expressions for the Angular Resolution of Compton Gamma-ray Detectors
}

D. M. Wright

February 5, 2004 


\section{Disclaimer}

This document was prepared as an account of work sponsored by an agency of the United States Government. Neither the United States Government nor the University of California nor any of their employees, makes any warranty, express or implied, or assumes any legal liability or responsibility for the accuracy, completeness, or usefulness of any information, apparatus, product, or process disclosed, or represents that its use would not infringe privately owned rights. Reference herein to any specific commercial product, process, or service by trade name, trademark, manufacturer, or otherwise, does not necessarily constitute or imply its endorsement, recommendation, or favoring by the United States Government or the University of California. The views and opinions of authors expressed herein do not necessarily state or reflect those of the United States Government or the University of California, and shall not be used for advertising or product endorsement purposes.

\section{Auspices Statement}

This work was performed under the auspices of the U.S. Department of Energy by University of California, Lawrence Livermore National Laboratory under Contract W-7405-Eng-48. 


\title{
Analytic Expressions for the Angular Resolution of Compton Gamma-ray Detectors
}

\author{
Douglas Wright \\ High Energy Physics Group, N Division \\ Lawrence Livermore National Laboratory
}

September 23, 2003

Revised January 9, 2004

\section{Introduction}

This paper describes the derivation of analytic expressions for the angular resolution of reconstructing gamma rays detected via Compton interactions. We consider two types of gamma-ray detectors: Compton-ring and electron-tracking devices.

In Compton-ring devices, the direction of the scattered electron is not resolved, only the total energy (electron and scattered photon) and the scattered photon direction are measured. The measured quantities define a cone about the axis of the scattered photon direction (see Figure 1). The initial photon direction lies along this cone. Thus for single events there is a ring-like ambiguity in the photon direction. By combining multiple events, the intersection of the reconstructed rings will resolve the initial direction of the photon source. In this paper, we derive the resolution of the cone angle for individual rings.

Electron-tracking type devices resolve the electron path. Although the scattered electron subsequently undergoes multiple-Coulomb scattering, it is possible to measure the initial electron direction with sufficiently high tracking resolution. By measuring the direction and energy of the electron and the direction of the scattered photon, the initial photon direction can be uniquely determined. The challenge for this type of detector is achieving the high tracking resolution.

In Section 2 we derive the well-known Compton formula for Compton-ring devices, an analytic expression for the angular resolution of the cone angle, and discuss the limits for applying the error formula. In Section 3 we repeat the derivation of the error function for the algebraically more complicated electron-tracking device. In the final section (Section 4) we derive the effect of position measurement error on the angular resolution, which applies to both detector types. All of the analytic results are cross-checked against empirical fits to a simple Monte Carlo simulation (Sections 2.5, 3.3, and 4.2).

Doppler broadening, the effect due to the initial (and intrinsically unknown) momentum of the atomic electron, can be ignored for gamma rays with initial energy greater than a few hundred $\mathrm{keV}$. For all kinematic calculations in this paper, the electron is taken to be initially at rest. 


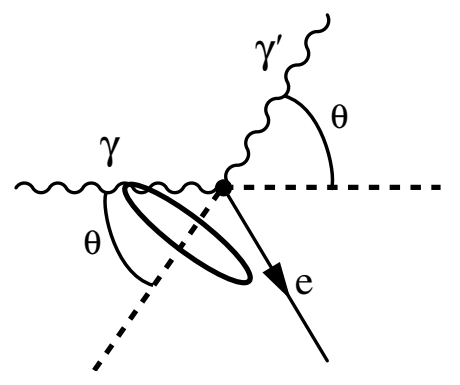

Figure 1: Compton interaction in which an incoming photon $(\gamma)$ scatters off of an atomic electron. Unless the electron direction is resolved, there is a $\phi$-like ambiguity (represented by the ring in the drawing) for the reconstructed initial photon direction.

\section{Compton-ring devices}

\subsection{Derivation of the Compton formula}

Assume that the initial electron is at rest, then from conservation of momentum, the electron recoils from the scattered photon (see Figure 1):

$$
\vec{p}_{e}=\vec{p}_{\gamma}-\vec{p}_{\gamma^{\prime}}
$$

Taking the square of the momentum and using $E_{\gamma}=p_{\gamma}$ and $E_{\gamma^{\prime}}=p_{\gamma^{\prime}}$ (since photons are massless and we are using units where $c=1$ ), results in

$$
p_{e}^{2}=E_{\gamma}^{2}+E_{\gamma^{\prime}}^{2}-2 E_{\gamma} E_{\gamma^{\prime}} \cos \theta .
$$

Using the invariant mass relation $p_{e}{ }^{2}=E_{e}{ }^{2}-m_{e}{ }^{2}$ and conservation of energy $\left(E_{\gamma}+m_{e}=E_{e}+E_{\gamma^{\prime}}\right)$ to eliminate the electron variables $p_{e}$ and $E_{e}$ results in

$$
\begin{array}{r}
E_{\gamma^{\prime}}+2 m_{e} E_{\gamma}+m_{e}{ }^{2}-2 E_{\gamma} E_{\gamma^{\prime}}-2 m E_{\gamma^{\prime}}+E_{\gamma^{\prime}}{ }^{2}-m_{e}{ }^{2} \\
=E_{\gamma^{\prime}}{ }^{2}+E_{\gamma^{\prime}}{ }^{2}-2 E_{\gamma} E_{\gamma^{\prime}} \cos \theta,
\end{array}
$$

which reduces to

$$
m_{e}\left(E_{\gamma}-E_{\gamma^{\prime}}\right)=E_{\gamma} E_{\gamma^{\prime}}(1-\cos \theta) .
$$

This is often rewritten as

$$
\cos \theta=1+\frac{m_{e}}{E_{\gamma}}-\frac{m_{e}}{E_{\gamma^{\prime}}}
$$

\subsection{Compton formula implications}

As the electron kinetic energy, $K_{e}=E_{e}-m_{e}$, approaches zero the scattering angle $\theta$ also goes to zero. Obviously at zero angle there is no scattering and the outgoing photon has the same energy as the incoming photon $\left(E_{\gamma}=E_{\gamma^{\prime}}\right)$. 
At the other extreme, the maximum electron energy (and minimum outgoing photon energy) occurs at $\theta=\pi$, i.e., when the photon scatters back in the direction opposite to that of the incoming photon. The maximum electron kinetic energy is

$$
K_{e}(\max )=\frac{E_{\gamma}^{2}}{E_{\gamma}+m_{e} / 2}
$$

while the minimum outgoing photon energy is not zero, but is instead

$$
E_{\gamma^{\prime}}(\min )=\frac{m_{e}}{2+m_{e} / E_{\gamma}}=\frac{1}{2} K_{e}(\max )\left(\sqrt{2 m_{e} / K_{e}(\max )+1}-1\right) .
$$

\subsection{Derivation of Compton angle error}

The Compton angle $\theta$ in Equation 1 depends only on the energy of the incoming $\left(E_{\gamma}=E_{\gamma^{\prime}}+E_{e}-m_{e}\right)$ and outgoing $\left(E_{\gamma^{\prime}}\right)$ gamma rays. If one considers only small Gaussian errors for the observables $E_{e}$ and $E_{\gamma^{\prime}}$, then one can derive an analytic expression for the error on $\theta$ as follows.

Taking the differential of Equation 1 yields

$$
d(\cos \theta)=-\sin \theta d \theta=-\frac{m_{e}}{E_{\gamma}{ }^{2}} d E_{\gamma}+\frac{m_{e}}{E_{\gamma^{\prime}}{ }^{2}} d E_{\gamma^{\prime}},
$$

which can be rewritten as

$$
d \theta=\frac{m_{e}}{\sin \theta E_{\gamma}{ }^{2}}\left[d E_{\gamma}-\frac{E_{\gamma}{ }^{2}}{E_{\gamma^{\prime}}{ }^{2}} d E_{\gamma^{\prime}}\right] .
$$

Since $E_{\gamma}=E_{e}+E_{\gamma^{\prime}}-m_{e}$, we have $d E_{\gamma}=d E_{e}+d E_{\gamma^{\prime}}$. Applying this to the previous equation for $d \theta$ results in

$$
d \theta=\frac{m_{e}}{\sin \theta E_{\gamma}^{2}}\left[d E_{e}+\left(1-\frac{E_{\gamma^{2}}{ }^{2}}{E_{\gamma^{\prime}}}\right) d E_{\gamma^{\prime}}\right] .
$$

This equation defines the partial derivatives $\partial \theta / \partial E_{e}$ and $\partial \theta / \partial E_{\gamma^{\prime}}$. So for Gaussian errors $\delta E_{e}$ and $\delta E_{\gamma^{\prime}}$, the error estimate for $\theta$ is

$$
\delta \theta=\frac{m_{e}}{\sin \theta E_{\gamma}{ }^{2}} \sqrt{\delta E_{e}{ }^{2}+\left(1-\frac{E_{\gamma}{ }^{2}}{{E_{\gamma^{\prime}}}^{2}}\right)^{2} \delta E_{\gamma^{\prime}}{ }^{2}} .
$$

This equation is valid as long as the fractional errors $\left(\delta E_{e} / E_{e}\right.$ and $\left.\delta E_{\gamma^{\prime}} / E_{\gamma^{\prime}}\right)$ are not too large. Furthermore, one must be careful when applying this equation in situations near $\theta=0$ or $\pi$.

\subsection{Caveats in error function near $\theta=0$ or $\pi$}

The case where $\theta$ is exactly zero is not a problem since this only occurs when the electron kinetic energy $\left(K_{e}=E_{e}-m_{e}\right)$ is zero. Thus there is no detected first interaction and one would not apply the Compton formula. Arbitrarily small values of $K_{e}$ also do not cause a problem for Equation 3 until they approach the magnitude of the energy resolution itself. 
For arbitrarily small values of the kinetic energy, i.e., $K_{e} / E_{\gamma} \ll 1$, the Compton formula (Equation 1) and energy conservation $\left(E_{\gamma^{\prime}}=E_{\gamma}-K_{e}\right)$ imply

$$
\sin ^{2} \theta \simeq \frac{2 m_{e} K_{e}}{E_{\gamma}^{2}}
$$

to lowest order in $K_{e} / E_{\gamma}$. Thus to lowest order, the square of Equation 3 can be approximated as

$$
\delta \theta^{2} \simeq \frac{m_{e}}{2 E_{\gamma}{ }^{2}}\left[\frac{\delta K_{e}^{2}}{K_{e}}+\frac{4 K_{e}}{E_{\gamma}{ }^{2}} \delta E_{\gamma^{\prime}}{ }^{2}\right] .
$$

From this one can see that $\delta \theta^{2}$ begins to blow up when the the kinetic energy becomes smaller than the square of the energy resolution $\delta K_{e}$, but is well behaved otherwise. Note that the second term $\left(\delta E_{\gamma^{\prime}}{ }^{2}\right)$ is suppressed for small $K_{e}$. When $K_{e}$ is smaller than about twice the energy resolution, the Gaussian approximation itself breaks down and Equation 3 will begin to overestimate the error in $\theta$. To apply this formula, one should impose a lower cutoff on the electron energy based on the energy resolution.

For the case where $\theta$ is near $\pi$, the scattered photon energy is near its minimum (but can not be zero - see Section 2.2). Because of finite detector resolution, the observed photon energy can fluctuate downward and even be lower than the minimally allowed energy. These kinematically unallowed events will be explicitly rejected since the Compton formula itself fails. However, the case where the photon energy reaches its minimum is kinematically allowed. For these events at or very near the minimum, the error formula approaches infinity. To highlight the behavior near the minimum energy, we can rewrite the equation using $E_{\gamma^{\prime}}=E_{\gamma^{\prime}}(\min )(1+\epsilon)$, where $\epsilon \ll 1$. In this limit, the Compton formula (Equation 1) implies

$$
\sin ^{2} \theta \simeq \frac{2 \epsilon m_{e}}{E_{\gamma}}
$$

Thus to lowest order, the square of Equation 3 can be approximated as

$$
\delta \theta^{2} \simeq \frac{m_{e}}{2 \epsilon E_{\gamma}{ }^{3}}\left[\delta E_{e}{ }^{2}+\left(1-\frac{(1-2 \epsilon) E_{\gamma}{ }^{2}}{E_{\gamma^{\prime}}{ }^{2}(\min )}\right)^{2} \delta E_{\gamma^{\prime}}{ }^{2}\right] .
$$

Neither term $\left(\delta E_{e}\right.$ or $\left.\delta E_{\gamma^{\prime}}\right)$ is suppressed, so in order to avoid this infinity (when $\epsilon \rightarrow 0$ ), one should eliminate events that are less than at least one standard deviation (in terms of the energy resolution) away from the minimum photon energy.

\subsection{Numerical cross-check of Compton-ring error function}

To check the validity of Equation 3 we performed a simple Monte Carlo analysis by generating Gaussian distributions for the input variables $E_{e}$ and $E_{\gamma^{\prime}}$ and compared the resulting distribution of $\theta$ with the analytic expression. We generated 100,000 events where the Compton electron kinetic energy $\left(E_{e}-m_{e}\right)$ was sampled from a Gaussian distribution with a mean of $100 \mathrm{keV}$ and RMS of $1 \mathrm{keV}$, and the scattered gamma ray energy distribution had a mean of $400 \mathrm{keV}$ with an RMS of $2 \mathrm{keV}$.

Using Equation 1 we reconstruct the Compton angle $\theta$ from the simulated observables. Figure 2 shows the reconstructed $\theta$ distribution. We fit a simple Gaussian function to this distribution. The parameters from the fit along with the results from the analytic expressions (Equations 1 and 3) are given in Table 1 The agreement between the fit and the analytic expression is excellent. 


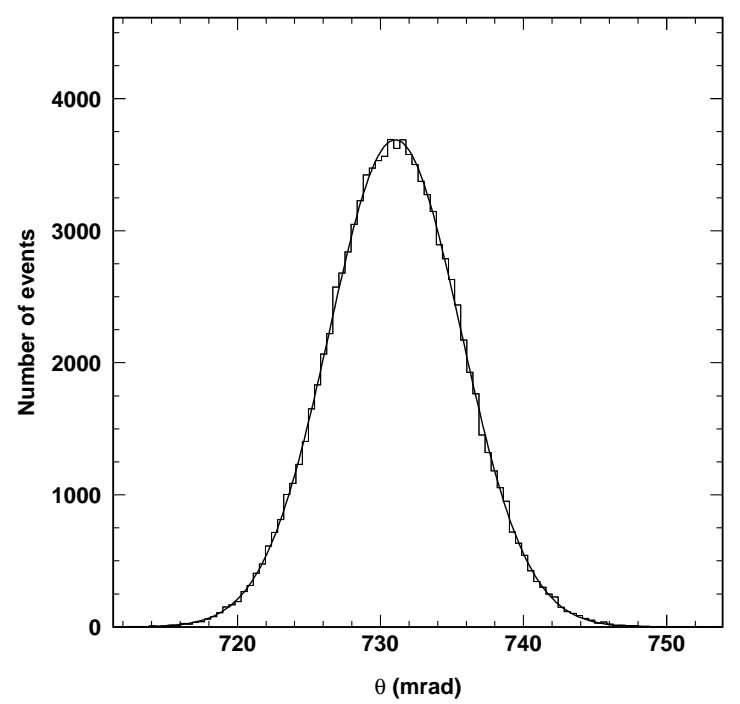

Figure 2: Compton-ring detector. Reconstructed Compton angle $\theta$ from a simulation of a single physical event sampled 100,000 times with Gaussian errors for the observed energies. The fit result is superimposed on the distribution.

Table 1: Compton-ring detector. Compton angle and error comparison between a fit to the simulation and an analytic calculation. Sample of 100,000 events where the electron kinetic energy is $100 \mathrm{keV}$ with an RMS error of $1 \mathrm{keV}$, and scattered gamma-ray energy of $400 \mathrm{keV}$ with an RMS error of $2 \mathrm{keV}$.

\begin{tabular}{|r|r|r|}
\hline Variable & Fit Result & Analytic Result \\
\hline$\theta(\mathrm{mrad})$ & $731.03 \pm 0.02$ & 731.01 \\
$\delta \theta(\mathrm{mrad})$ & $4.61 \pm 0.01$ & 4.61 \\
\hline
\end{tabular}




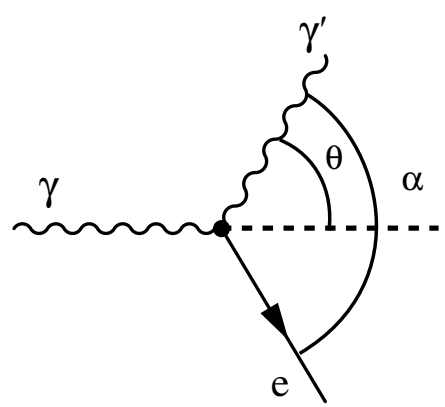

Figure 3: Compton interaction. Same as Figure 1 with the scattered photon and electron opening angle labeled as $\alpha$.

\section{Electron-tracking devices}

\subsection{Derivation of kinematic relations (à la Compton formula)}

The kinematic relations derived in Section 2 also apply to the electron-tracking type detector. Since the electron-tracking devices resolve the direction of both the electron and photon, it is useful to derive some additional relations in terms of the opening angle $\alpha$ (defined in Figure 3 ) of the observed particles. From conservation of momentum $\vec{p}_{\gamma}=\vec{p}_{\gamma^{\prime}}+\vec{p}_{e}$ and using $E_{\gamma}=p_{\gamma}$ and $E_{\gamma^{\prime}}=p_{\gamma^{\prime}}$ (since photons are massless), the square of the initial photon momentum is

$$
E_{\gamma}{ }^{2}=E_{\gamma^{\prime}}{ }^{2}+p_{e}{ }^{2}+2 E_{\gamma^{\prime}} p_{e} \cos \alpha .
$$

Using the invariant mass relation $p_{e}{ }^{2}=E_{e}{ }^{2}-m_{e}{ }^{2}$ and conservation of energy $\left(E_{\gamma}=E_{\gamma^{\prime}}+E_{e}-m_{e}\right)$ to eliminate the incoming photon energy $E_{\gamma}$ from the previous equations gives

$$
E_{\gamma^{\prime}}{ }^{2}+2 E_{\gamma^{\prime}}\left(E_{e}-m_{e}\right)+\left(E_{e}-m_{e}\right)^{2}=E_{\gamma^{\prime}}{ }^{2}+p_{e}{ }^{2}+2 E_{\gamma^{\prime}} p_{e} \cos \alpha .
$$

Solving for $E_{\gamma^{\prime}}$ results in

$$
E_{\gamma^{\prime}}=\frac{m_{e}\left(E_{e}-m_{e}\right)}{E_{e}-m_{e}-p_{e} \cos \alpha}
$$

Applying conservation of energy $\left(E_{\gamma}=E_{\gamma^{\prime}}+E_{e}-m_{e}\right)$ to the above equation gives

$$
E_{\gamma}=\frac{\left(E_{e}-m_{e}\right)\left(E_{e}-p_{e} \cos \alpha\right)}{E_{e}-m_{e}-p_{e} \cos \alpha} .
$$

Note that the ratio of Equation 4 and 5 is

$$
\frac{E_{\gamma}}{E_{\gamma^{\prime}}}=\frac{E_{e}-p_{e} \cos \alpha}{m_{e}}
$$

Combing the Compton formula (Equation 1) with Equations 4 and 5 yields a relation between the Compton angle $\theta$ and the angle between the electron and scattered photon $\alpha$ :

$$
\cos \theta=1-\frac{\left(E_{e}-m_{e}-p_{e} \cos \alpha\right)^{2}}{\left(E_{e}-m_{e}\right)\left(E_{e}-p_{e} \cos \alpha\right)} .
$$




\subsection{Derivation of electron-tracking angular error function}

The error function derived in Section 2 also applies for electron tracking devices with one important distinction: since the electron trajectory is measured, there is no ambiguity in determining the direction of the initial photon. One component of the photon direction is completely determined by the plane formed by the scattered electron and scattered photon. The error on the angular component within that plane is calculated below.

We start with the analytic expression for the angular error $\mathrm{d} \theta$ given by Equation 2. For electron tracking devices we measure the electron energy $\left(E_{e}\right)$ but not the scattered photon energy $\left(E_{\gamma^{\prime}}\right)$. Using Equation 4 we can find the relationship between the error on $E_{\gamma^{\prime}}$ with the error on the measured quantities $E_{e}$ and $\alpha$. We can then combine this relation with the previously derived error function to find an equation in terms of measured quantities only.

Taking the differential of Equation 4 gives

$$
d E_{\gamma^{\prime}}=\frac{m_{e} d E_{e}}{E_{e}-m_{e}-p_{e} \cos \alpha}-\frac{m_{e}\left(E_{e}-m_{e}\right)}{\left(E_{e}-m_{e}-p_{e} \cos \alpha\right)^{2}}\left[d E_{e}-\cos \alpha d p_{e}+p_{e} \sin \alpha d \alpha\right]
$$

Using $E_{e} d E_{e}=p_{e} d p_{e}$ (which follows from $E_{e}{ }^{2}=p_{e}{ }^{2}-m_{e}{ }^{2}$ ) and Equations 4 and 5 to simplify the expression, we get

$$
d E_{\gamma^{\prime}}=\left(\frac{E_{\gamma^{\prime}}}{E_{e}-m_{e}}-\frac{E_{\gamma^{\prime}}^{2}\left(p_{e}-E_{e} \cos \alpha\right)}{m_{e} p_{e}\left(E_{e}-m_{e}\right)}\right) d E_{e}-\frac{E_{\gamma^{\prime}}{ }^{2} p_{e} \sin \alpha}{m_{e}\left(E_{e}-m_{e}\right)} d \alpha
$$

Substituting this into Equation 2 and regrouping in terms of $\mathrm{d} E_{e}$ and $\mathrm{d} \alpha$ gives

$$
d \theta=\frac{\left(E_{\gamma}{ }^{2}-E_{\gamma^{\prime}}{ }^{2}\right)}{E_{\gamma}{ }^{2}\left(E_{e}-m_{e}\right) \sin \theta}\left[\left(1-\frac{m_{e}}{E_{\gamma^{\prime}}}-\frac{E_{e}}{p_{e}} \cos \alpha+\frac{m_{e}\left(E_{e}-m_{e}\right)}{E_{\gamma}{ }^{2}-E_{\gamma^{\prime}}{ }^{2}}\right) d E_{e}+p_{e} \sin \alpha d \alpha\right] .
$$

This equation defines the partial derivatives $\partial \theta / \partial E_{e}$ and $\partial \theta / \partial \alpha$. So for Gaussian errors $\delta E_{e}$ and $\delta \alpha$, the error estimate for $\theta$ is

$$
\delta \theta=\frac{\left(E_{\gamma}{ }^{2}-E_{\gamma^{\prime}}{ }^{2}\right)}{E_{\gamma}{ }^{2}\left(E_{e}-m_{e}\right)^{2} \sin \theta} \sqrt{\left(1-\frac{m_{e}}{E_{\gamma^{\prime}}}-\frac{E_{e}}{p_{e}} \cos \alpha+\frac{m_{e}\left(E_{e}-m_{e}\right)}{E_{\gamma}{ }^{2}-E_{\gamma^{\prime}}{ }^{2}}\right)^{2} \delta E_{e}{ }^{2}+p_{e}{ }^{2} \sin ^{2} \alpha \delta \alpha^{2}} .
$$

This equation is valid as long as the fractional errors in the measured quantities are not too large. As in the Compton-ring case, one must be careful when applying this error function near $\theta=0$ or $\pi$. Also note that this is just the error of one component of the angle. The orthogonal component and its associated error are completely determined by the plane defined by the observed electron and photon trajectories.

\subsection{Numerical cross-check of electron-tracking error function}

To check the validity of Equation 7 we performed a simple Monte Carlo analysis by generating Gaussian distributions for the input variables $E_{e}$ and $\alpha$ and compared the resulting distribution of $\theta$ with the analytic expression. We generated 100,000 events where the Compton electron kinetic energy $\left(E_{e}-m_{e}\right)$ was sampled from a Gaussian distribution with a mean of $1000 \mathrm{keV}$ and RMS of $10 \mathrm{keV}$, 


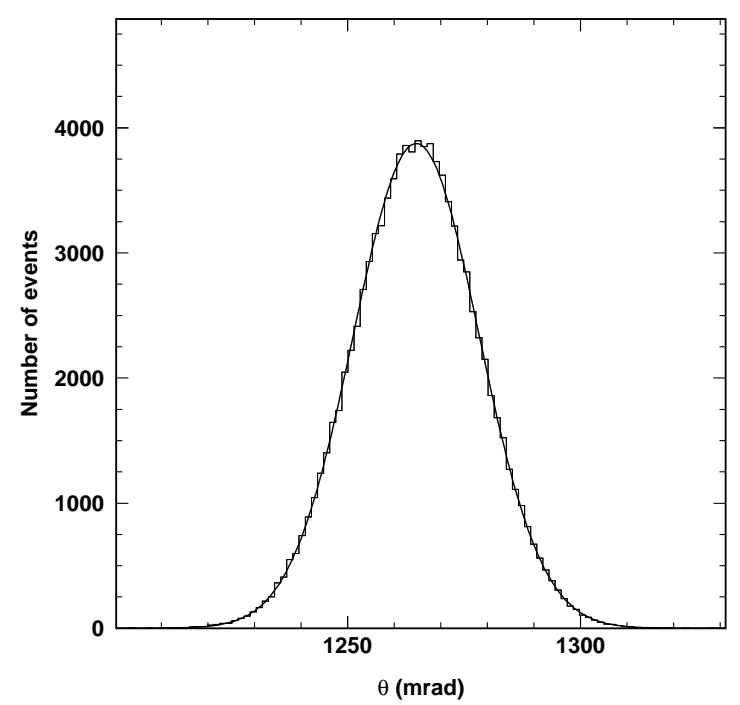

Figure 4: Reconstructed Compton angle $\theta$ from a simulation of a single physical event sampled 100,000 times with Gaussian errors for the observed energies. The fit result is superimposed on the distribution.

Table 2: Electron-tracking detector. Compton angle and error comparison between a fit to simulation and an analytic calculation. Sample of 100,000 events where the Electron kinetic energy is $1000 \mathrm{keV}$ with an RMS error of $10 \mathrm{keV}$, and the opening angle between the scattered electron and gamma ray is $1600 \mathrm{mrad}$ with an RMS of $10 \mathrm{mrad}$. with and RMS error of $2 \mathrm{keV}$.

\begin{tabular}{|r|r|r|}
\hline Variable & Fit Result & Analytic Result \\
\hline$\theta(\mathrm{mrad})$ & $1264.70 \pm 0.04$ & 1264.75 \\
$\delta \theta(\mathrm{mrad})$ & $13.47 \pm 0.03$ & 13.49 \\
\hline
\end{tabular}

and the opening angle between the scattered electron and gamma ray had a mean of 1600 mrad with an RMS of 10 mrad.

Using Equation 7 we reconstruct the angle $\theta$ from the simulated observables. Figure 4 shows the reconstructed $\theta$ distribution. We fit a simple Gaussian function to this distribution. The parameters from the fit along with the results from the analytic expressions (Equations 6 and 7) are given in Table 2. The agreement between the fit and the analytic expression is excellent. 


\section{Generic effects}

\subsection{Derivation of angular error due to position resolution}

The direction of the outgoing photon is determined from the measured positions of the two interaction points. The measurement uncertainty of these positions creates an associated uncertainty in the direction of the photon. One can derive an analytic expression for the angular error due to the position resolution as follows.

If two points $v_{1}=\left(x_{1}, y_{1}, z_{1}\right), v_{2}=\left(x_{2}, y_{2}, z_{2}\right)$ define the direction of the outgoing photon $\vec{v}=v_{2}-v_{1}$, then the angle between the photon and some arbitrary unit vector $\hat{n}$ is

$$
\hat{n} \cdot \vec{v}=v \cos \theta=v_{\|},
$$

where $v_{\|}$is the component of $\vec{v}$ parallel to $\hat{n}$. The component perpendicular to $\hat{n}$ is then $v_{\perp}=v \sin \theta$. Taking the differential of $v \cos \theta=v_{\|}$gives

$$
d v \cos \theta-\sin \theta v d \theta=d v_{\|} .
$$

Using $v d v=v_{\perp} d v_{\perp}+v_{\|} d v_{\|}$(which comes from the differential of $v^{2}=v_{\|}^{2}+v_{\perp}^{2}$ ) and substituting into the equation above, results in

$$
\cos \theta\left(\frac{v_{\perp}}{v} d v_{\perp}+\frac{v_{\|}}{v} d v_{\|}\right)-\sin \theta v d \theta=d v_{\|}
$$

Using the $\sin \theta$ and $\cos \theta$ relations, this can be simplified to

$$
d \theta=\frac{1}{v^{2}}\left(v_{\|} d v_{\perp}-v_{\perp} d v_{\|}\right) .
$$

For Gaussian measurement errors, this gives an error estimate of

$$
\delta \theta=\frac{1}{v^{2}} \sqrt{v_{\|}^{2} \delta v_{\perp}^{2}+v_{\perp}^{2} \delta v_{\|}^{2}} \cdot
$$

If one wants the error estimator for just the unit vector parallel to the photon direction, i.e., $\hat{n}=\vec{v} / v$, then the above equation reduces to $\delta \theta=\delta v / v$.

If $\delta d$ is the measurement error for each coordinate $(x, y, z)$ of each point $v_{1}$ and $v_{2}$, then the measurement error for the coordinates of the vector $\vec{v}$ are

$$
\delta v_{\perp}=\delta v_{\|}=\sqrt{2} \delta d
$$

Then Equation 8 simplifies to

$$
\delta \theta=\frac{\sqrt{2}}{v} \delta d .
$$

This relation holds as long as the separation between the two interaction points $v$ is significantly larger than the measurement resolution $\delta d$. 


\subsection{Numerical cross-check of error function due to position resolution}

To check the validity of Equation 9 we performed a simple Monte Carlo analysis by generating Gaussian distributions for the individual coordinates of the two interaction points that define the photon direction. We generated 100,000 events where each coordinate had an RMS of $0.01 \mathrm{~mm}$. The mean starting and ending locations were $(0,0,0)$ and $(0,0,1) \mathrm{mm}$.

For each event we calculate the opening angle between the photon direction and the expected direction. Figure 4 shows the opening angle $\theta$ distribution. We fit a simple Gaussian times a $\sin \theta$ function (to account for the geometric solid angle effect) to this distribution. The parameters from the fit along with the results from the analytic expression (Equation 9) are given in Table 3. The agreement between the fit and the analytic expression is excellent. 


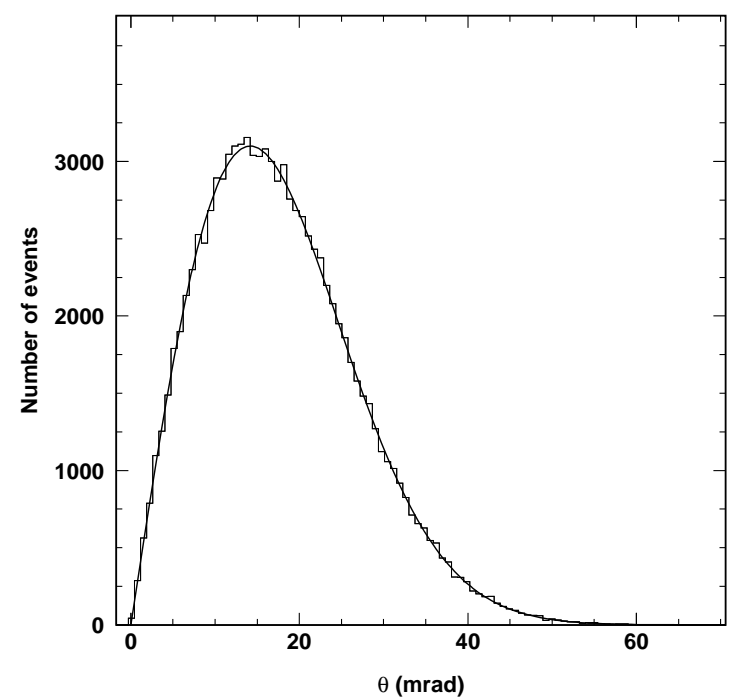

Figure 5: Angular deviation of the reconstructed photon direction from a simulation of a single physical event sampled 100,000 times with Gaussian errors for the measured interaction locations. The fit result is superimposed on the distribution.

Table 3: Opening angle comparison between the analytic calculation and the fit to the simulation. Sample of 100,000 events where the starting and ending coordinates have an RMS error of $0.01 \mathrm{~mm}$. The separation between the coordinates is $1.00 \mathrm{~mm}$

\begin{tabular}{|r|r|r|}
\hline Variable & Fit Result & Analytic Result \\
\hline$\theta(\mathrm{mrad})$ & $0.16 \pm 0.49$ & 0.00 \\
$\delta \theta(\mathrm{mrad})$ & $14.10 \pm 0.17$ & 14.14 \\
\hline
\end{tabular}

